\title{
ON SCALAR AND VECTOR COVARIANTS OF LINEAR ALGEBRAS*
}

\author{
BY \\ OLIVE C. HAZLETT
}

\section{INTRODUCTION}

1. Relation to the literature. This paper concerns itself with two rather different kinds of covariants of the general linear algebra, which might, for convenience, be distinguished by the adjectives scalar and vector. Consider the general linear algebra $\dagger E$ with the units $e_{1}, \cdots, e_{n}$ and with the constants of multiplication $\gamma_{i j k}(i, j, k=1, \cdots, n)$, where the general number of the algebra is $X=\sum x_{i} e_{i}$. In a previous paper $\ddagger$ we defined a rational integral covariant $C$ of the algebra $E$ as a rational integral function of the $\gamma$ 's and the $x$ 's which possesses the invariantive property whenever the units are subjected to a linear transformation. The paper just mentioned uses such covariants to characterize linear associative algebras in two and three units. The present paper proves for such covariants several fundamental theorems analogous to the fundamental theorems in the theory of invariants for algebraic forms. Since these covariants are isobaric and homogeneous, we can apply here Hilbert's proof of the "finiteness" of the number of covariants of algebraic forms.

We must not, however, content ourselves with the study of such covariants, since they are not sufficient to characterize linear algebras, both associative and non-associative, even when there are only two units. Accordingly, we consider rational integral functions of the $\gamma$ 's, the $x$ 's and also the units $e_{i}(i=1, \cdots, n)$ which possess the invariantive property. Since these functions involve the $e$ 's, we call them vector covariants in contradistinction to the functions $C$ mentioned above, which we might call scalar covariants. At first sight, it might seem as if we were confronted with a rather inconvenient difficulty for the following reason. In a theory of such covariants we would expect to find treated certain differential operators analogous to the familiar annihilators $\Omega$ and 0 ; but Scheffers has shown that, in a linear algebra, a

* Part I was presented to the Society December 27, 1917, under a different title; Part II was presented February 23, 1918.

$\dagger$ For the fundamental definitions in the theory of linear algebras, see Section 2. The most familiar linear algebras are number fields (in particular, the field of reals and the field of ordinary complex numbers) and quaternions.

$\ddagger$ A n n als of Mathematic s, second series, vol. 16 (1914), pp. 1-6. 
derivative is not uniquely determined unless multiplication is commutative, and we are concerned with algebras both commutative and non-commutative. This Gordian knot can, however, be readily cut by a device. We can, accordingly, derive the annihilators in the approved manner, and hence show that every rational integral vector covariant of the linear algebra is a covariant of the general number of the algebra $X=\sum x_{i} e_{i}$ and a suitable set of scalar covariants of the algebra. From this fact flow theorems analogous to those prowed for scalar covariants and, in particular, the "finiteness" of vector covariants.

2. Definitions. A linear algebra $E$ over the field $F$ is a set of hypercomplex numbers of the form $X=\sum_{i=1}^{n} x_{i} e_{i}$, where the coördinates $x_{i}$ range independently over $F$. Here the units $e_{i}$ are such that $e_{i} e_{j}=\sum_{k=1}^{n} \gamma_{i j k} e_{k}$ $(i, j=1, \cdots, n)$, where (i) the constants of multiplication $\gamma_{i j k}$ are in $F$, (ii) the sum of two numbers $X$ and $Y$ of the algebra is $X+Y=$ $\sum_{i=1}^{n}\left(x_{i}+y_{i}\right) e_{i}$ and (iii) numbers of the algebra combine under addition and multiplication according to the distributive law. Unless explicitly stated, we do not assume the commutative nor the associative law of multiplication.*

If we have a number $X=\sum x_{i} e_{i}$ of an algebra whose coördinates are (ordinary) complex numbers, then we can find a number $Y=\sum y_{i} e_{i} \neq 0$ and a scalar $\omega$ such that $X Y=\omega Y$ if and only if the right-hand characteristic determinant $\delta(\omega) \equiv\left|\sum_{i=1}^{n} \gamma_{i j k} x_{i}-d_{j k} \omega\right|$ is zero. Here $d_{j j}=1, d_{j k}=0$ if $k \neq j$. Similarly, there is a number $Y \neq 0$ and a scalar $\omega$ such that $Y X=\omega Y$ if and only if the left-hand characteristic determinant

vanishes.

$$
\delta^{\prime}(\omega) \equiv\left|\sum_{i=1}^{n} \gamma_{j i k} x_{i}-d_{j k} \omega\right|
$$

For convenience in the study of covariants of a linear algebra, we introduce the notion of weight. If we subject the algebra $E$ to the transformation

$$
\begin{array}{ll}
e_{r}^{\prime}=\lambda e_{r} & (\lambda \neq 0) \\
e_{i}^{\prime}=e_{i} & (i \neq r),
\end{array}
$$

the $\gamma^{\prime}$ 's are subject to the induced transformation where $\gamma_{i j k}$ is unaltered when $i, j, k \neq r, \gamma_{i r}(i, j \neq r)$ is multiplied by $\lambda^{-1}, \gamma_{r i r}$ and $\gamma_{i r r}(i \neq r)$ are unaltered, $\gamma_{r r r}$ is multiplied by $\lambda, \gamma_{r j k}$ and $\gamma_{j r k}(j, k \neq r)$ by $\lambda$ and $\gamma_{r r k}(k \neq r)$ by $\lambda^{2}$. Hence we shall say that $\gamma_{i j k}(i, j, k \neq r)$ is of weight $0, \gamma_{i j r}(i, j \neq r)$ of weight -1 in $e_{r}, \gamma_{r i r}$ and $\gamma_{i r r}(i \neq r)$ of weight $0, \gamma_{r r r}$ of weight $1, \gamma_{r j k}$ and $\gamma_{j r k}(j, k \neq r)$ of weight 1 and $\gamma_{r r k}(k \neq r)$ of weight 2 in $e_{r}$. For every unit $e_{r}$ we thus assign to $\gamma_{i j k}$ a weight with respect to that unit, which is counted in the following way: the first subscript, $i$, contributes +1 if $i=r$, other-

\footnotetext{
* For definitions of a linear associative algebra by a set of independent postulates, see Dickson, these Trans a ctions, vol. 4 (1903), pp. 21-26.
} 
wise 0 ; the second, $j$, contributes +1 if $j=r$, otherwise 0 ; and the third, $k$, contributes -1 if $k=r$, otherwise 0 . The sum of these three partial weights is called the weight of $\gamma_{i j k}$ with respect to $e_{r}$.

For a similar reason, we shall say that $e_{r}$ is of weight 1 in $e_{r}$ but of weight 0 in any other unit $e_{i}(i \neq r)$.

If $X=\sum x_{i} e_{i}$ is the general number of the algebra $E$, then if $x_{1}^{\prime}, \cdots, x_{n}^{\prime}$ are the coördinates of $X$ when it is expressed in terms of the new units-that is, $X=\sum x_{i}^{\prime} e_{i}^{\prime}$-then $x_{r}^{\prime}=x_{r} / \lambda$ and $x_{i}^{\prime}=x_{i}(i \neq r)$. For this reason.one might be tempted to say that $x_{r}$ is of weight -1 with respect to $e_{r}$ and that the other $x$ 's are of weight 0 with respect to $e_{r}$. In the applications, however, it will be better so to define weight that every $x$ has a weight which is positive or zero with respect to any unit $e_{r}$. Accordingly, we shall agree that $x_{r}$ is of weight 0 in $e_{r}$ and that $x_{i}(i \neq r)$ is of weight 1 in $e_{r}$.

We shall use the term total wisht of a term for the sum of the weights of that term in all the units $e_{1}, \cdots, e_{n}$. Notice that the total weight of any $\gamma, x_{i}$ or $e_{i}$ is 1 , and thus the total weight of a product of a number of $\gamma$ 's, $x$ 's and $e$ 's is the degree of that term.

If $P$ is the product of a number of $\gamma$ 's (or a number of $e$ 's) such that it is of weight $w_{r}$ in a particular unit, $e_{r}$, it is multiplied by $\lambda^{v_{r}}$ under the transformation $(\lambda)_{r}$. If, however, $P$ is the product of a number of $x$ 's such that it is of degree $s$ in the $x$ 's and of weight $w_{r}$ in $e_{r}$, then under the transformation $(\lambda)_{r}$ it is multiplied by $\lambda^{w_{r}-s}$.

Any polynomial in the $\gamma$ 's, the $x$ 's and the $e$ 's such that all terms have the same weight with respect to a particular unit $e_{r}$ will be said to be isobaric with respect to $e_{r}$. If the terms have the same total weight in all the units, the polynomial will be said to be isobaric on the whole. If $P$ is a polynomial in the $\gamma$ 's, the $x$ 's and the $e$ 's which is isobaric with respect to $e_{r}$, of weight $w_{r}$ in $e_{r}$ and which is homogeneous in the $x$ 's of degree $s$, it is multiplied by $\lambda^{w_{r}-s}$ under the transformation $(\lambda)_{r}$.

\section{Part I. Scalar Covariants}

3. Some fundamental theorems. The invariancy of $I$ under the transformation

$T:$

$$
\begin{aligned}
& e_{l}^{\prime}=\sum_{m=1}^{n} a_{l m} e_{m} \quad(l=1, \cdots, n), \\
& A=\left|a_{l m}\right| \neq 0
\end{aligned}
$$

is expressed by the formula

$$
I\left(\gamma_{i j k}^{\prime}\right)=\phi\left(a_{l m}\right) I\left(\gamma_{i j k}\right)
$$

where the $\gamma_{i j k}$ are the constants of multiplication of the original algebra and 
the $\gamma_{i j k}^{\prime}$ are the constants of multiplication of the transformed algebra. Here $I\left(\gamma_{i j k}^{\prime}\right)$ has been written for $I\left(\gamma_{111}^{\prime}, \gamma_{112}^{\prime}, \cdots, \gamma_{n n n}^{\prime}\right), \phi\left(a_{l m}\right)$ for $\phi\left(a_{11}, a_{12}, \cdots, a_{n n}\right)$ and $I\left(\gamma_{i j k}\right)$ for $I\left(\gamma_{111}, \gamma_{112}, \cdots, \gamma_{n n n}\right)$. Hence by applying the special transformation $e_{i}^{\prime}=\lambda e_{i}(\lambda \neq 0 ; i=1, \cdots, n)$, it follows that $I$ is homogeneous in the $\gamma$ 's.

Under this transformation $T$ the $\gamma_{i j k}$ are subject to an induced transfoimation such that

$$
\sum_{p} \gamma_{i j p}^{\prime} a_{p q}=\sum_{k, l} a_{i k} a_{j l} \gamma_{k l q} \quad(i, j, q=1, \cdots, n) .
$$

If we keep $i$ and $j$ fixed in (2) and let $q$ range from 1 to $n$, we have $n$ nonhomogeneous linear equations in the $n$ unknowns $\gamma_{i j p}^{\prime}$ which can be solved uniquely, since $A \neq 0$. Hence each $\gamma_{i j p}^{\prime}$ is a homogeneous function of degree $n+1$ in the $a$ 's divided by $A$.

Accordingly, if $I\left(\gamma_{i j k}\right)$ is a rational integral invariant of total weight $w$ in the units and hence homogeneous of degree $w$ in the $\gamma^{\prime} \mathrm{s}, I\left(\gamma_{i j k}^{\prime}\right)$ is a rational integral homogeneous function of degree $(n+1) w$ in the $a_{l m}$ divided by $A^{w}$. But, since $I\left(\gamma_{i j k}\right)$ is independent of the $a$ 's , this implies that $\phi\left(a_{l m}\right)$ is a rational integral homogeneous function $\psi\left(a_{l m}\right)$ of degree $(n+1) w$ in the $a$ 's divided by $A^{w}$. That is,

$$
I\left(\gamma_{i j k}^{\prime}\right)=\frac{\psi\left(a_{l m}\right)}{A^{w}} I\left(\gamma_{i j k}\right)
$$

for every transformation of determinant $A \neq 0$. Hence under the inverse transformation $T^{-1}$

$$
I\left(\gamma_{i j k}\right)=\frac{\psi\left(\frac{A_{m l}}{A}\right)}{\left(A^{-1}\right)^{w}} I\left(\gamma_{i j k}{ }^{\prime}\right),
$$

where $A_{m l}$ is the cofactor of $a_{m l}$. Combining these two results, we see that

$$
\psi\left(a_{l m}\right) \psi\left(\frac{A_{m l}}{A}\right)=1
$$

or, since $\psi$ is homogeneous of degree $(n+1) w$,

$$
\psi\left(a_{l m}\right) \psi\left(A_{m l}\right)=A^{(n+1) w} .
$$

But, since $A$ is irreducible, this can be true only if $\psi\left(a_{l m}\right)=k A^{u}$, where $k$ is some constant. Then $\psi\left(A_{m l}\right)=k A^{\prime u}$, where $A^{\prime}=\left|A_{m l}\right|=A^{n-1}$, and hence $u=w / n$. By applying the identical transformation, it is evident that $k=1$, and thus

$$
\phi\left(a_{l m}\right)=A^{w / n} .
$$

This last formula can be proved in the above manner familiar in the classical invariant theory, or it can also be proved for associative algebras by the 
following device. If $X=\sum x_{i} e_{i}$ is a number of the general $n$-ary linear algebra $E=\left(e_{1}, \cdots, e_{n}\right)$, then $E X=E=X E$. That is, if we multiply each unit $e_{i}$ of $E$ by $X$, we obtain $n$ linearly independent numbers of $E$ which can be taken as a new set of units. This remark still applies if we restrict ourselves to the general associative algebra. Accordingly, replace $e_{i}$ by $X e_{i}(i=1, \cdots, n)$. Then

$$
e_{i}^{\prime} e_{j}^{\prime} \equiv\left(X e_{i}\right)\left(X e_{j}\right)=\sum_{m}\left(\sum_{k, l} x_{k} \gamma_{k j l} \gamma_{i l m}\right)\left(X e_{m}\right) \quad(i, j=1, \cdots, n) .
$$

Thus $I\left(\gamma_{i j k}^{\prime}\right)$ is of degree $2 w$ in the $\gamma^{\prime}$ s. But $A^{r}$ is of degree $r n$ and $I\left(\gamma_{i j k}\right)$ is of degree $w$ in these $\gamma$ 's. Hence $2 w=r n+w$, or $r=w / n$.

Also, if $w_{i}$ is the weight of $I$ in $e_{i}$, then $w_{1}=w_{2}=\cdots=w_{n}$. This follows from equations (1) and (3) and the fact that $I$ must be unaltered (except possibly for sign) when any two units, say $e_{i}$ and $e_{j}$, are interchanged.

Since $w / n$ is the weight of $I$ in any particular unit, say $e_{1}, w / n$ is a positive integer. Notice, moreover, that $w$ is equal to the degree of $I$, since each $\gamma_{i j k}$ is of total weight 1 in the units. Thus we have

Theorem I. For an n-ary linear algebra $E=\left(e_{1}, p \cdots, e_{n}\right)$ with constants of multiplication $\gamma_{i j k}$, let $I$ be a rational integral invariant of degree d. Then $I$ is homogeneous and isobaric of total weight $d$ in all the units, and $d / n$ is a positive integer. If we subject the units of $E$ to the transformation $T$ of determinant $A \neq 0$, carrying $E$ into the algebra $E^{\prime}$ with constants of multiplication $\gamma_{i j k}^{\prime}$, then

$$
I\left(\gamma_{i j k}^{\prime}\right)=A^{d / n} I\left(\gamma_{i j k}\right) .
$$

The covariancy of the rational integral function $C$ under the transformation $T$ is expressed by the formula

$$
C\left(\gamma_{i j k}^{\prime} ; x_{l}^{\prime}\right)=\phi\left(a_{l m}\right) C\left(\gamma_{i j k} ; x_{l}\right) .
$$

Such a covariant is not necessarily homogeneous in the coördinates $x_{i}$ of the general number of the algebra, but it is the sum of covariants $C_{k}$ such that each $C_{k}$ is homogeneous in the $x$ 's. Accordingly, we may, without loss of generality, restrict ourselves to the study of homogeneous covariants. Every covariant $C$ which is homogeneous in the $x$ 's is also homogeneous in the $\gamma$ 's. If $C$ is homogeneous of degree $s$ in the $x$ 's and of degree $d$ in the $\gamma_{i j k}$, then $C\left(\gamma_{i j k}^{\prime} ; x_{l}^{\prime}\right)$ is a rational integral homogeneous function of degree $(n+1) d+(n-1) s$ in the $a$ 's, divided by $A^{d+s}$. Thus $\phi\left(a_{l m}\right)$ in this case is a rational integral homogeneous function, $\psi$, of the $a$ 's of degree $(n+1) d+(n-1) s$ divided by $A^{d+s}$, and consequently

$$
\psi\left(A_{m l}\right) \psi\left(a_{l m}\right)=A^{(n+1) d+(n-1) s} .
$$

Thence it follows that

$$
\phi\left(a_{l m}\right)=A^{(d-8) / n} .
$$


When we subject $E$ to the special transformation

$$
e_{i}^{\prime}=e_{i}(i \neq n), \quad e_{n}^{\prime}=\lambda e_{n}(\lambda \neq 0),
$$

each term of $C\left(\gamma_{i j k}^{\prime}\right)$ is equal to the corresponding term of $C\left(\gamma_{i j k}\right)$ multiplied by $\lambda^{w_{n}-8}$, where $w_{n}$ is the weight of that term in $e_{n}$. This follows at once from the remarks at the end of section 2 , since this is the transformation $(\lambda)_{n}$ 。 But under this transformation, $C\left(\gamma_{i j k}\right)$ is multiplied by $\lambda^{(d-s) / n}$ and thus $w_{n}-s=(d-s) / n$ or $w_{n}=[d+(n-1) s] / n$. It follows that the index of the power of $A$-namely $(d-s) / n$-is an integer. Moreover, it is not negative, for it is the weight of the coefficient of a term of $C$ which is independent of $x_{n}$. Now such a term actually occurs in $C$, as otherwise $x_{n}$ would be a factor of $C$, and hence (in view of the covariancy of $C$ ) every linear function of the $x$ 's would be a factor of $C$, which is impossible. Thus we have proved

Theorem II. For an n-ary linear algebra $E=\left(e_{1}, \cdots, e_{n}\right)$ where the constants of multiplication are $\gamma_{i j k}$ and the general number of the algebra is $X=\sum x_{i} e_{i}$, let $C$ be a rational integral covariant of degree $d$ in the $\gamma_{i j k}$ and degree $s$ in the $x_{i}$. Then $C$ is isobaric of total weight $d+(n-1) s$ in all the units. If $C$ is homogeneous in the $x$ 's, it is homogeneous in the $\gamma$ 's. Moreover, if we subject the units of $E$ to the transformation $T$ of determinant $A \neq 0$, carrying $E$ into the algebra $E^{\prime}$ with constants of multiplication $\gamma_{i j k}^{\prime}$, then

$$
C\left(\gamma_{i j k}^{\prime} ; x_{l}^{\prime}\right)=A^{(d-s) ! n} C\left(\gamma_{i j k} ; x_{l}\right) .
$$

The index, $(d-s) / n$, is an integer, positive or zero.*

4. Fundamenta! sets of invariants and covariants for $n=1,2$. When $n=1$, the multiplication table is $e_{1} e_{1}=\gamma e_{1}$ and the general number of the algebra is $x_{1} e_{1}$. Hence $\delta(\omega)=\delta^{\prime}(\omega)=\omega-\gamma x_{1}$; and the only rational integral invariants in this case are powers of $\gamma$, and the only rational integral scalar covariants of the unary algebra are all polynomials in $\gamma$ and $x_{1}$.

When $n=2$, the multiplication table is of the form $e_{i} e_{j}=\gamma_{i j 1} e_{1}+\gamma_{i j 2} e_{2}$ $(i, j=1,2)$ and the general number of the algebra is $X=x_{1} e_{1}+x_{2} e_{2}$. Hence

where

$$
\begin{aligned}
\delta(\omega) & =\omega^{2}-\omega\left[\Gamma_{1} x_{1}+\Gamma_{2} x_{2}\right]+\left[\Gamma_{5} x_{1}^{2}+\Gamma_{9} x_{1} x_{2}+\Gamma_{6} x_{2}^{2}\right], \\
\delta^{\prime}(\omega) & \left.=\omega^{2}-\omega\left[\Gamma_{3} x_{1}+\Gamma_{4} x_{2}\right]+\Gamma_{7} x_{1}^{2}+\Gamma_{10} x_{1} x_{2}+\Gamma_{8} x_{2}^{2}\right],
\end{aligned}
$$

$$
\begin{array}{ll}
\Gamma_{1}=\gamma_{111}+\gamma_{122}, & \Gamma_{2}=\gamma_{211}+\gamma_{222}, \\
\Gamma_{3}=\gamma_{111}+\gamma_{212}, & \Gamma_{4}=\gamma_{121}+\gamma_{222},
\end{array}
$$

* By the use of this theorem, we can readily prove the following corollary: Every rational integral invariant and covariant of the general linear algebra vanishes for a nilpotent algebra. This theorem was stated and proved for invariants in a paper in the A n n a ls of $\mathbf{M}$ a t h em a t i c s, second series, vol. 18 (1916), p. 84, and the proof for covariants proceeds in essentially the same way. 


$$
\begin{aligned}
& \Gamma_{5}=\gamma_{111} \gamma_{122}-\gamma_{112} \gamma_{121}, \quad \Gamma_{6}=\gamma_{211} \gamma_{222}-\gamma_{212} \gamma_{221}, \\
& \Gamma_{7}=\gamma_{111} \gamma_{212}-\gamma_{112} \gamma_{211}, \quad \Gamma_{8}=\gamma_{121} \gamma_{222}-\gamma_{122} \gamma_{221}, \\
& \Gamma_{9}=\gamma_{111} \gamma_{222}+\gamma_{211} \gamma_{122}-\gamma_{121} \gamma_{212}-\gamma_{112} \gamma_{221}, \\
& \Gamma_{10}=\gamma_{111} \gamma_{222}+\gamma_{121} \gamma_{212}-\gamma_{122} \gamma_{211}-\gamma_{112} \gamma_{221} .
\end{aligned}
$$

We may prove without any difficulty that, for a binary algebra, every homogeneous isobaric function of the constants of multiplication for which $w_{1}=w_{2}$ and which is annihilated by

$$
\begin{aligned}
U_{12}=\left(\gamma_{121}+\right. & \left.\gamma_{211}\right) \frac{\partial}{\partial \gamma_{111}}+\left(\gamma_{222}-\gamma_{121}\right) \frac{\partial}{\partial \gamma_{122}}+\left(\gamma_{222}-\gamma_{211}\right) \frac{\partial}{\partial \gamma_{212}} \\
& +\left[\left(\gamma_{212}+\gamma_{122}\right)-\gamma_{111}\right] \frac{\partial}{\partial \gamma_{112}}+\gamma_{221}\left[\frac{\partial}{\partial \gamma_{121}}+\frac{\partial}{\partial \gamma_{211}}-\frac{\partial}{\partial \gamma_{222}}\right]
\end{aligned}
$$

is an invariant of the algebra. We can prove a similar theorem for scalar covariants. Accordingly, one may readily determine the rational integral scalar covariants of a given weight, and so one might hope to find a fundamental set of covariants for the binary algebra.

One can, however, avoid such troubles in view of

TheOREM III. Every scalar covariant of the binary algebra $E=\left(e_{1}, e_{2}\right)$ where the constants of multiplication are $\gamma_{i j k}$ and where the general number of the algebra is $X=x_{1} e_{1}+x_{2} e_{2}$ is a covariant of the characteristic determinants $\delta$ and $\delta^{\prime}$.

We want to show that any rational integral function $C\left(\gamma_{i j k} ; x_{l}\right)$ of the eight $\gamma$ 's and the $x$ 's which has the invariantive property under the group of linear transformations on $e_{1}$ and $e_{2}$ is a function of $\Gamma_{1}, \cdots, \Gamma_{8}$ and the $x$ 's which is a covariant of the system of forms $\Gamma_{1} x_{1}+\Gamma_{2} x_{2}, \Gamma_{3} x_{1}+\Gamma_{4} x_{2}$, $\Gamma_{5} x_{1}^{2}+\Gamma_{9} x_{1} x_{2}+\Gamma_{6} x_{2}^{2}, \Gamma_{7} x_{1}^{2}+\Gamma_{10} x_{1} x_{2}+\Gamma_{8} x_{2}^{2}$ under the group of linear transformations on $x_{1}$ and $x_{2}$. Since $x_{1}$ and $x_{2}$ are contragredient to $e_{1}$ and $e_{2}, C\left(\gamma_{i j k} ; x_{l}\right)$ has the invariantive property under the group of linear transformations on $x_{1}$ and $x_{2}$ where the $\gamma$ 's are transformed according to (2). Thus we can prove our theorem if we can show that any function of the $\gamma$ 's is a function of $\Gamma_{1}, \cdots, \Gamma_{8}$. Now this follows from the fact that the Jacobian of $\Gamma_{1}, \cdots, \Gamma_{8}$ is

$$
J=\left|\begin{array}{llllllll}
1 & 0 & 0 & 0 & 0 & 0 & 0 & 1 \\
0 & 1 & 0 & 0 & 0 & 0 & 1 & 0 \\
1 & 0 & 0 & 0 & 0 & 1 & 0 & 0 \\
0 & 1 & 0 & 0 & 1 & 0 & 0 & 0 \\
\gamma_{122} & 0 & -\gamma_{121} & 0 & -\gamma_{112} & 0 & 0 & \gamma_{111} \\
0 & \gamma_{211} & 0 & -\gamma_{212} & 0 & -\gamma_{221} & \gamma_{222} & 0 \\
\gamma_{212} & 0 & -\gamma_{211} & 0 & 0 & \gamma_{111} & -\gamma_{112} & 0 \\
0 & \gamma_{121} & 0 & -\gamma_{122} & \gamma_{222} & 0 & 0 & -\gamma_{221}
\end{array}\right|,
$$


which is not identically zero, since the terms free of $\gamma_{112}, \gamma_{111}, \gamma_{222}$ and $\gamma_{221}$ equal the four-rowed determinant in the lower left-hand corner.

5. Finiteness of the rational, integral scalar covariants. In view of the homogeneity and isobarism of rational integral scalar covariants, we can readily prove that they are all expressible as polynomials in a finite subset. For, by Hilbert's theorem about an infinite sequence of polynomials, * any such covariant $C$ can be expressed in the form

$$
C(\gamma ; x)=\sum K_{j}(\gamma ; x) C_{j}(\gamma ; x)
$$

where the $C_{j}$ are scalar covariants of the algebra which are the same for every $C$. Since $C$ and $C_{j}$ are homogeneous and isobaric, we may suppose that the $K_{j}$ are also. If we subject $E$ to the transformation $T$ of determinant $A \neq 0$, (5) is replaced by

$$
A^{w} C(\gamma ; x)=\sum A^{w_{j}} K_{j}\left(\gamma^{\prime} ; x^{\prime}\right) C_{j}(\gamma ; x),
$$

where $C$ is of index $w$ and $C_{j}$ is of index $w_{j}$. Now operate on both sides of (6) with

$$
\Omega_{a} \equiv \sum\left( \pm i_{1} \cdots i_{n}\right) \frac{\partial^{n}}{\partial a_{1 i_{1}} \cdots \partial a_{n i_{n}}}
$$

until both sides of the resulting equation no longer involve the $a$ 's. Then, by a well-known theorem of Hilbert's (which is essentially one given by Mertens and Gordan $\dagger$ ), (6) becomes

$$
N C(\gamma ; x)=\sum N_{j} C_{j}^{\prime}(\gamma ; x) C_{j}(\gamma ; x),
$$

where the $C_{j}^{\prime}$ are scalar covariants of the algebra, and $N$ and $N_{j}$ are constants different from zero. Thus we prove by induction

Theorem IV. For the general n-ary linear algebra there is a finite set $C_{1}$, $\cdots, C_{m}$ of scalar covariants such that every rational integral scalar covariant of the algebra is a polynomial in $C_{1}, \cdots, C_{m}$.

Corollary. For the general $n$-ary linear algebra there is a finite set $B_{1}, \cdots, B_{p}$ of rational scalar covariants such that every rational integral scalar covariant of the algebra is a covariant of this set of algebraic forms. For convenience, such a set of covariants will be called basic.

* "Ueber die Theorie der algebraischen Formen," Mathematis che Annalen, vol. 36 (1890), p. 474.

$\dagger$ Hilbert, loc. cit., p. 524; Gordan, Beveis, dass jede Covariante und Invariante einer binären Form eine ganze Function mit numerischen Coefficienten einer endlichcn Anzahl solcher Formen ist, J o u rn a l f ür M a the mat i k, vol. 69 (1868), py. 323-354; Die simultanen Systeme binärer Formen, M a the mat is c h e Annalen, vol. 2 (1870), pp. 227-280; Invariantentheorie (1887), vol. II, §9; Mertens, Beweis dass alle Invarianten und Covarianten . . ., J o urnal f ür M a the matik, vol. 100 (1887), pp. 223-230. 


\section{Part II. Vector Covariants}

6. Preliminary remarks. As we pointed out in the introduction, scalar covariants are not sufficient to characterize linear algebras-in fact, they are not sufficient to characterize potent algebras in 2 units, for the two algebras

$$
\begin{array}{lll}
e_{1} e_{1}=e_{1}, & e_{1} e_{2}=e_{2} e_{1}=0, & e_{2} e_{2}=\gamma e_{1} \quad(\gamma>0) ; \\
e_{1} e_{1}=e_{1}, & e_{1} e_{2}=e_{2} e_{1}=0, & e_{2} e_{2}=0
\end{array}
$$

are not equivalent with respect to the field of reals, and yet for both algebras $\delta=\delta^{\prime}=\omega\left(\omega-x_{1}\right)$ and both have the same rank and the same index.

Accordingly we shall now consider vector covariants. By a rational integral function of the $\gamma$ 's, the $x$ 's and the $e$ 's we shall understand any linear combination of a finite number of products of units of the algebra where the coefficients are polynomials in the $\gamma$ 's and $x$ 's. It must be borne in mind that, since multiplication is not in general commutative nor associative, we cannot, for instance, combine into one term two such as $e_{1}\left[\left(e_{1} e_{2}\right) e_{3}\right]$ and $\left(e_{1} e_{1}\right)\left(e_{2} e_{3}\right)$. That is, in some terms the multiplication is to be carried out in one order and in other terms in a different order. Every rational integral vector covariant $V$ is, however, the sum of a finite number of vector covariants $V_{k}$ such that, in any one $V_{k}$, the multiplication indicated in every term is to be carried out in the same order.

7. Fundamental properties. We can readily show that if a vector covariant is homogeneous in the units, $e_{i}$, and homogeneous in the $x_{i}$, it is homogeneous in the $\gamma_{i j k}$. Moreover it is isobaric in any unit $\boldsymbol{e}_{i}$, and its weight in $e_{j}$ is equal to its weight in $e_{i}(i, j=1, \cdots, n)$. From these properties we can readily prove the analogue of Theorem II, which we state here merely for the sake of completeness.

Theorem V. For an n-ary linear algebra $E=\left(e_{1}, \cdots, e_{n}\right)$ where the constants of multiplication are $\gamma_{i j k}$ and the general number of the algebra is $X=\sum x_{i} e_{i}$, let $V$ be a rational integral vector covariant of degree $d$ in the $\gamma_{i j k}$, of degree $s$ in the $x$ 's and of degree $v$ in the e's. Then $V$ is isobaric of weight $w=d+(n-1) s+v$ in the units altogether. If $V$ is homogeneous in the $x$ 's and the e's, it is homogeneous in the $\gamma$ 's. Furthermore, if we subject the units of $E$ to the transformation $T$ of determinant $A \neq 0$, carrying $E$ into the algebra $E^{\prime}=\left(e_{1}^{\prime}, \cdots, e_{n}^{\prime}\right)$ where the general number of the algebra is $X^{\prime}=\sum x_{i}^{\prime} e_{i}^{\prime}$ and the constants of multiplication are $\gamma_{i j k}^{\prime}$, then

$$
V\left(\gamma_{i j k}^{\prime} ; x_{l}^{\prime} ; e_{m}^{\prime}\right)=A^{(d-s+v) / n} V\left(\gamma_{i j k} ; x_{l} ; e_{m}\right) .
$$

Moreover the index $(d-s+v) / n$ is an integer, positive or zero.

8. Annihilators for vector covariants. Since we are dealing with a linear algebra, every vector covariant whose degree in the units is greater than 2 
can be made linear in the units. Henceforth, unless otherwise specified, we shall think of every vector covariant as linear in the units.

First consider absolute vector covariants of degree 1 in the $x$ 's. Now such a covariant is of the form

$$
\sum_{i=1}^{n}\left[x_{1} \phi_{1 i}(\gamma)+x_{2} \phi_{2 i}(\gamma)+\cdots+x_{n} \phi_{n i}(\gamma)\right] e_{i}
$$

where each $\phi_{i i}$ is a polynomial of weight zero in each unit, and each $\phi_{i j}(i \neq j)$ is a polynomial of weight 1 in $e_{i}$, of weight -1 in $e_{j}$, and of weight zero in the other units. But any polynomial in the $\gamma$ 's of degree $d$ is of total weight $d$ in the $e$ 's altogether. Hence each $\phi_{i j}=0(i \neq j)$ and each $\phi_{i i}$ is a constant, $c_{i}$. But, since (7) is unaltered if we interchange any two units, the $c_{i}$ are all equal, and thus a vector covariant which is of the first degree in the $x$ 's is necessarily a constant multiple of the general number of the algebra.

Next, consider absolute vector covariants of degree 2 in the $x$ 's ; such a covariant is necessarily of the form

$$
\sum_{i=1}^{n}\left[x_{i}^{2} \phi_{i i i}(\gamma)+x_{i} \sum_{j \neq i} x_{j} \phi_{i j i}(\gamma)+\sum_{j, k \neq i} x_{j} x_{k} \phi_{j k i}(\gamma)\right] e_{i}
$$

where each $\phi_{i i i}$ is of weight 1 in $e_{i}$ and of weight zero in $e_{l}(l \neq i)$, each $\phi_{i i i}(i \neq j)$ is of weight 1 in $e_{j}$ and of weight zero in $e_{l}(l \neq j)$, and each $\phi_{j k i}(j, k \neq i)$ is of weight -1 in $e_{i}$, of weight 1 in $e_{j}$ and in $e_{k}$ and of weight zero in $e_{l}(l \neq i, j, k)$. Accordingly each $\phi$ is of degree 1 in the $\gamma$ 's. In particular,

$$
\begin{aligned}
& \phi_{111}=\alpha \gamma_{111}+\beta\left(\gamma_{122}+\gamma_{133}+\gamma_{1 n n}\right)+\gamma\left(\gamma_{212}+\cdots+\gamma_{n 1 n}\right), \\
& \phi_{121}=\delta \gamma_{121}+\epsilon\left(\gamma_{323}+\cdots+\gamma_{n 2 n}\right)+\zeta \gamma_{211}+\eta\left(\gamma_{233}+\cdots+\gamma_{2 n n}\right)+\theta \gamma_{222}, \\
& \phi_{231}=\kappa \gamma_{231}+\lambda \gamma_{321}, \quad \phi_{221}=\mu \gamma_{221},
\end{aligned}
$$

and any $\phi_{i i i}$ is obtained from $\phi_{111}$ by interchanging the subscripts 1 and $i$; any $\phi_{i j i}(j \neq i)$ is obtained from $\phi_{121}$ by interchanging 1 and $i$, and 2 and $j$; and so forth. Hence the difference between (8) and $\beta C_{n-1} X+\gamma C_{n-1}^{\prime} X$ $+(\alpha-\beta-\gamma) X^{2}$ is a covariant of the type (8) where the $\alpha, \beta, \gamma$ are zero. Here $C_{n-1}$ and $C_{n-1}^{\prime}$ are the coefficients of $\omega^{n-1}$ in $\delta(\omega)$ and $\delta^{\prime}(\omega)$ respectively. Hence consider the covariant (8) where $\alpha, \beta, \gamma$ are all zero. Since such a covariant must be unaltered under the transformation

$$
e_{1}^{\prime}=e_{1}+e_{2}, \quad e_{i}=e_{i}(i \neq 1),
$$

we must have $\delta=\epsilon=\zeta=\eta=\theta=\kappa=\lambda=\mu=0$. Therefore every ra- 
tional integral absolute vector covariant of degree 2 in the $x$ 's is a homogeneous polynomial of degree 2 in $C_{n-1}^{\prime}, C_{n-1}^{\prime}$ and $X=\sum x_{i} e_{i}$.

More generaliy we have

Theorem VI. Every rational integral vector covariant of the n-ary linear algebra is a covariant of the general number of the algebra and any basic set of scalar covariants of the algebra.

We prove this by using a set of annihilators for vector covariants-differential operators analogous to the familiar Aronhold operators $\Omega$ and 0 . In deriving such operators for the scalar covariants, we used Taylor's theorem with a remainder for polynomials in the $\gamma$ 's and the $x$ 's, and so we now seem to be confronted with the necessity of inquiring if this theorem (or one closely analogous) holds for polynomials in the $\gamma$ 's, the $x$ 's and the $e$ 's-which we might, for brevity, call vector polynomials. This brings forward the question of a differential calculus for such vector polynomials.

Now Scheffers* has considered the theory of functions of hypercomplex variables. In a linear algebra over the field $C$ of ordinary complex numbers in which division is generally possible, he considers functions of the form $f=\sum f_{i} e_{i}$ where each $f_{i}$ is an analytic function of the $x_{j}(j=1, \cdots, n)$. When

$$
\begin{aligned}
d x & =\sum_{i} d x_{i} e_{i}, \\
\text { aif } & =\sum_{i, j} \frac{\partial f_{i}}{\partial x_{k}} d x_{k} e_{i} .
\end{aligned}
$$

Then Scheffers shows that such a linear algebra which possesses a principal unit permits of functions $f$ with derivatives with respect to $x$ which are independent of $\Delta x_{1}, \cdots, \Delta x_{n}$-that is, independent of the "direction" in which $\Delta x$ approaches zero-if and only if multiplication is commutative.

It would seem, then, as if we could not hope to find annihilators for vector covariants of the general $n$-ary linear algebra. There are, however, several ways of avoiding this little difficulty.

As we pointed out at the beginning of this sect on, we can think of a vector covariant as linear in the $e$ 's ; and so, to derive the desired annihilators, we need only to consider such derivatives as

$$
\frac{\partial e_{1}}{\partial e_{1}}, \frac{\partial e_{2}}{\partial e_{1}}, \cdots
$$

With this end in view, let us consider the linear function $\phi\left(e_{1}\right)=e_{1}$. Re-

*Verallgemeinerung der Grundlagen der gewöhnlichen complexen Functionen, L e i p z i g e r B e ri c h t e, vol. 45 (1893), pp. 828-848; vol. 46 (1894), pp. 120-134. There are abstracts of these articles in Paris C o in p te s Re $\mathrm{r}_{\mathrm{d}} \mathrm{u}$ s, vol. 116 (1893), pp. 1114-1117, 1242-1244. 
placing $e_{1}$ by $e_{1}+\Delta e_{1}$, where the increment $\Delta e_{1}$ is a scalar multiple of $e_{2}$, say $m e_{2}$, we have $\Delta \phi=\Delta e_{1}$. Now divide on the right by $\Delta e_{1}$, i. e. find a number $u$ such that $u \cdot \Delta e_{1}=\Delta e_{1}$. If we are dealing with any particular algebra, this number $u$ depends on that algebra; but we are interested in the general algebra, and thus we want a number $u$ such that $u \cdot \Delta e_{1}=\Delta e_{1}\left(\right.$ or $\left.u \cdot e_{2}=e_{2}\right)$ for every $n$-ary linear algebra. One such number $u$ is the scalar 1 . If we take $u=1, d e_{1} / d e_{1}=1$. With a similar understanding,

$$
\begin{aligned}
& \frac{\partial e_{2}}{\partial e_{1}}=0, \\
& \frac{\partial e_{3}}{\partial e_{1}}=0, \quad \cdots .
\end{aligned}
$$

Similarly for the derivatives with respect to the other units.

The advantage of defining these derivatives in this way is that we can differentiate with respect to any unit, such as $e_{1}$, in a purely formal mannerprecisely as if we were differentiating with respect to a scalar. Thus a vector covariant which is linear in the units is annihilated by the differential operators

$$
U_{i j}+\sum\left(\frac{\partial x_{k}^{\prime}}{\partial a_{i j}}\right) \cdot \frac{\partial}{\partial x_{k}}+\sum\left(\frac{\partial e_{k}^{\prime}}{\partial a_{i j}}\right) \cdot \frac{\partial}{\partial e_{k}} \quad(i, j=1, \cdots, n),
$$

where the $U_{i j}$ are the annihilators for the invariants of the $n$-ary algebra.

Or we may reason as follows.

Every vector covariant when linear in the units has these annihilators, since such a covariant has the invariantive property when the $e$ 's are replaced by a set of cogredient scalars.

Theorem VI now follows at once from the fact that the annihilators for the vector covariants of the algebra are the same as those for covariants of a basic set of scalar covariants of the algebra and the general number of the algebra, $\sum x_{i} e_{i}$.

9. Finiteness of vector covariants. We now readily prove

Theorem VII. For the general n-ary linear algebra there is a finite set $V_{1}$, $\cdots, V_{m}$ of rational integral vector covariants such that every vector covariant of the algebra is a linear function of $V_{1}, \cdots, V_{m}$.

For every vector covariant of a linear algebra may be made linear in the $e$ 's . Now thinking of the $e$ 's as replaced by a set of cogredient scalars, which we shall call the $y$ 's, we have a set of rational integral functions of the variables $x_{i}(i=1, \cdots, n)$ and the contragredient variables $y_{i}(i=1, \cdots, n)$ together with the $n^{3}$ parameters $\gamma_{i j k}\left(i_{i j}, k=1, \cdots, n\right)$ which are homogeneous and isobaric and which have the invariantive property under the total linear 
group on the $n$ variables $x_{i}$. Accordingly Hilbert's “finiteness" proof applies. Or this theorem can be proved as a corollary of Theorem VI. Mount Holyoxm Columer, Bouth Haduey, Mass. 